\title{
Effects of Manufacturing Process Variables on the Tablet Weight Variation of Mini-tablets Clarified by a Definitive Screening Design
}

\author{
Shui Usuda, ${ }^{a}$ Naohiro Masukawa, ${ }^{b}$ Kok Hoong Leong, ${ }^{c}$ Kotaro Okada, ${ }^{a}$ and Yoshinori Onuki*,a \\ ${ }^{a}$ Laboratory of Pharmaceutical Technology, Graduate School of Medicine and Pharmaceutical Science for Research, \\ University of Toyama; 2630 Sugitani, Toyama 930-0194, Japan: ${ }^{b}$ JMP Japan, SAS Institute Japan Ltd.; 6-10-1 \\ Roppongi, Minato-ku, Tokyo 106-6111, Japan: and ${ }^{c}$ Department of Pharmaceutical Chemistry, Faculty of Pharmacy, \\ University of Malaya; 50603 Kuala Lumpur, Malaysia. \\ Received May 18, 2021; accepted June 22, 2021
}

This study investigated the effect of manufacturing process variables of mini-tablets, in particular, the effect of process variables concerning fluidized bed granulation on tablet weight variation. Test granules were produced with different granulation conditions according to a definitive screening design (DSD). The five evaluated factors assigned to DSD were: the grinding speed of the sample mill at the grinding process of the active pharmaceutical ingredient (X1), microcrystalline cellulose content in granules (X2), inlet air temperature (X3), binder concentration (X4) and the spray speed of the binder solution (X5) at the granulation process. First, the relationships between the evaluated factors and the granule properties were investigated. As a result of the DSD analysis, the mode of action of granulation parameters on the granule properties was fully characterized. Subsequently, the variation in tablet weight was examined. In addition to mini-tablets $(3 \mathrm{~mm}$ diameter), this experiment assessed regular tablets $(8 \mathrm{~mm}$ diameter). From the results for regular tablets, the variation in tablet weight was affected by the flowability of granules. By contrast, regarding the mini-tablets, no significant effect on the variation of tablet weight was found from the evaluated factors. From this result, this study further focused on other important factors besides the granulation process, and then the effect of the die-hole position of the multiple-tip tooling on tablet weight variation was proven to be significant. Our findings provide a better understanding of manufacturing mini-tablets.

Key words mini-tablet; tablet weight variation; granule property; wet granulation; fluidized bed granulator; definitive screening design

\section{Introduction}

Mini-tablets, which are small tablets with a diameter ranging from 1.5 to $4.0 \mathrm{~mm}$, have recently been gaining much attention, in particular for use in pediatric medicines. ${ }^{1)}$ The small size has a great advantage in terms of swallowability: it allows child patients to swallow easily. Indeed, several reports indicate that mini-tablets show superior swallowability by child patients compared with other dosage forms such as syrup. $^{2,3)}$ In addition, as far as pediatric medicine is concerned, the dose of the medicine is required to change drastically according to the age and body weight of the patient. Mini-tablets have a great capacity for dose adjustment: the designated dose can be easily prepared by varying the number of tablets. ${ }^{1)}$ Furthermore, mini-tablets can be manufactured in the same method as regular tablets: rotary tableting machines are generally used for the production of mini-tablets. ${ }^{1)}$ From this issue, appropriate product qualities (e.g., uniform size and shape, smooth surface, low porosity and high strength) are ensured by comparing with other multiparticulate dosage forms such as granules. Of course, active pharmaceutical ingredients (APIs) in mini-tablets are much more stable than those in liquid formulations because mini-tablets are a solid dosage form by nature. There are many attractive properties, in particular for pediatric medication; however, to date, knowledge of mini-tablet manufacturing is still insufficient. In particular, know-how of manipulating tablet weight uniformity is in great demand.

With the spread of the concept of quality-by-design (QbD) into the pharmaceutical industries, a detailed understanding of the relationships between the factors and the characteristics is required for pharmaceutical product development. $\mathrm{QbD}$ is a modern concept in the design of pharmaceutical products, ${ }^{4,5)}$ which was introduced in the International Conference on Harmonization Q8 guideline in 2008. .) According to the QbD concept, the quality of a pharmaceutical product must be ensured by the formulation and the manufacturing processes. To meet the concept, QbD strongly recommends applying the methodology of design of experiments (DOE) to pharmaceutical product development.

Definitive screening design (DSD) is a new class of DOE method incorporating a three-level experimental design. ${ }^{7,8}$ DSD has the unique capacity for evaluating the pure-quadratic effects of various factors on characteristics, as well as the main effects of such factors and their two-factor interactions. DSD also has a great advantage in the aspect of reducing the number of experimental runs. DSD uses the conference matrix to produce the experimental design. The conference matrix possesses fold-over pairs corresponding to each evaluated factor and overall center points. Because of this feature, the total number of experimental runs required is only about twice the number of the evaluated factors. This contributes to significant reductions in terms of time, resources and cost during pharmaceutical development. Furthermore, DSD can create response surfaces integrating quadratic effects of factors even though the size of experimental data is small. To date, DSD has been used in a wide range of pharmaceutical formulation 
studies to screen crucial manufacturing process variables. The dosage forms include oral disintegrating films, ${ }^{9)}$ modifiedrelease drug products, ${ }^{10)}$ intranasal gel formulations, ${ }^{11)}$ semisolid dosage forms ${ }^{12,13)}$ and lyophilized products. ${ }^{14)}$ Recently, we have investigated the usefulness of DSD for use in the QbD approach through a case study on the formulation design of ethenzamide-containing orally disintegrating tablet. As a consequence of the study, we demonstrated that DSD could be applied to develop pharmaceuticals in accordance with the QbD concept.

The purpose of the present study was to gain a better understanding of the effect of manufacturing process variables on the weight variation of mini-tablets. In particular, this study featured the effect of the wet granulation process. The wet granulation process is known to have a significant impact on the properties of the resulting tablets by manipulating the granule's properties via modifying the powder properties. ${ }^{15-18)}$ In addition, there are several reports that powder properties (particle size, powder density, flowability and others) affected the weight variation of mini-tablets. ${ }^{19-21)}$ However, systematic knowledge concerning the weight variation of mini-tablets is yet to be obtained. From these perspectives, the present study investigated the relationships among process variables of fluidized bed granulation, granule properties and weight variation of mini-tablets. Acetaminophen was chosen as a model API for this study because of its wide use in pediatric patients. We have investigated the effect of acetaminophen on the tablet properties (e.g., disintegration time and tensile strength) in the formulation study of regular tablets. ${ }^{22,23)}$ The acetaminophencontaining granules were prepared by changing the level of the evaluated factors according to DSD, and then their effect on the properties of the granules and tablet weight variation were analyzed using DSD. In addition to mini-tablets, regular tablets of $8 \mathrm{~mm}$ diameter were prepared in the present study to understand the tablet weight variation in more detail. Furthermore, in the course of the above experiments, this study also examined the tablet weight variation induced by differences in the die-hole position of the multiple-tip tooling used in manufacturing minitablets, because it was suggested that its location might be a crucial factor. As a consequence, this study could provide valuable information on the production of mini-tablets with high tablet weight uniformity.

\section{Experimental}

Materials Acetaminophen was kindly donated by Watahan Trading (Tokyo, Japan). Microcrystalline cellulose (MCC, Ceolus PH-101; Asahi Kasei Chemicals, Tokyo, Japan), lactose monohydrate (LAC, Pharamatose 200M; DFE Pharma, Goch, Germany), low-substituted hydroxypropyl cellulose (L-HPC, LH-21; Shin-Etsu Chemical, Tokyo, Japan), hydroxypropyl cellulose (HPC, HPC-L; Nippon Soda, Tokyo, Japan) and magnesium stearate (MgSt; FUJIFILM Wako, Osaka, Japan) were purchased from the indicated suppliers.

Wet Granulation API-containing granules were prepared by the wet granulation process with a fluidized bed granulator (FD-MINI; Powrex, Hyogo, Japan). It was ground by a sample mill (NARA Machinery, Tokyo, Japan) with three different rotational speeds $(7000,9000$ and $11000 \mathrm{rpm})$. Regarding the wet granulation process, the designated amounts of API, LAC and MCC were mixed for $3 \mathrm{~min}$ using a polyethylene bag. Then, the powder blend was granulated with the fluidized bed granulator. The airflow rate was adjusted to $0.15-0.20 \mathrm{~m}^{3} / \mathrm{min}$, and the spray air flow rate was set at $20 \mathrm{~L} / \mathrm{min}$. An aqueous solution of 4,7 or $10 \%$ HPC-L was used as the binder solution. The API, L-HPC and HPC-L contents were fixed at 25, 7 and $3 \mathrm{~g}$ in $98 \mathrm{~g}$ of the resulting granules. The amounts of LAC and MCC were varied according to DSD: the total content of the mixture was composed of 63 in $98 \mathrm{~g}$ of the resulting granules. The levels of the evaluated factors for each granule were assigned using DSD. The experimental designs were created using the statistical software JMP ${ }^{\circledR}$ Pro 15 (SAS Institute, Cary, NC, U.S.A.).

Preparation of Test Tablets The prepared granules were sieved through a $710 \mu \mathrm{m}$ mesh screen. Next, lubricant was added to the granules to adjust the concentration to $2 \%$ of the resulting tablet weight and then mixed for $1 \mathrm{~min}$ using a polyethylene bag. The lubricated granules were compressed into a mini-tablet ( $3 \mathrm{~mm}$ in diameter) and a regular tablet $(8 \mathrm{~mm}$ in diameter), using a tableting machine (VELA5 0312SS2MZ; Kikusui Seisakusho, Kyoto, Japan) with a gravity feeder system. For manufacturing the mini-tablets, a standard concave multiple-tip tooling (6 tips per die; I Holland, Nottingham, England) was installed at one station on the rotational disk. Except for the experiment to examine the effect of the hole position of multiple-tip tooling on tablet weight variation, tableting process of the test tablets was performed with rotational speed of the rotating disk in the rotary tableting machine at $10 \mathrm{rpm}$. As for the preparation of the mini-tablets used in the experiment to examine the effect of the hole position, the rotation speed was set at $5 \mathrm{rpm}$ in order to separately collect the resulting mini-tablets produced from individual die holes. The designated weights of the mini-tablet and regular tablet were set at 15 and $180 \mathrm{mg}$, respectively (the mini-tablet weight was set at $1 / 12$ of the regular tablet weight). The composition of the resulting tablets was as follows: the weight ratio of API/sum of LAC and MCC/L-HPC/HPC-L/ $\mathrm{MgSt}=25 / 63 / 7 / 3 / 2$.

Angle of Repose The sample granule was allowed to fall onto a base with a diameter of $80 \mathrm{~mm}$ through a glass funnel to form a cone. The angle of the slope of the cone was measured as the angle of repose (AR).

Compressibility Index The compressibility index (CI) of the mannitol powders was calculated from the bulk density $\left(\rho_{\text {bulk }}\right)$ and tapped density $\left(\rho_{\text {tapped }}\right)$ as measured using a multitester MT1001 (Seishin Enterprise, Tokyo, Japan). About $90 \mathrm{~mL}$ of the test granules were placed into a $100 \mathrm{~mL}$ graduated cylinder, and the weight of the powder was measured. The $\rho_{\text {bulk }}$ was calculated as the ratio of the mass of the untapped granule sample and its volume. The sample granule in the cylinder was tapped by the multitester, and then $\rho_{\text {tapped }}$ was calculated. The CI value was calculated from the values of $\rho_{\text {bulk }}$ and $\rho_{\text {tapped }}$ as follows:

$$
\mathrm{CI}(\%)=\frac{\rho_{\text {tapped }}-\rho_{\text {bulk }}}{\rho_{\text {tapped }}} \times 100
$$

Dispersion Index The dispersion index (DI) of the granules was also measured using the multitester. The test granules $(10 \mathrm{~g})$ were dropped from the top of a cylinder with a length of $60 \mathrm{~cm}$ and diameter of $10 \mathrm{~cm}$ to a shallow glass dish with a diameter of $10 \mathrm{~cm}$. After that, the weight of the granules remaining on the dish was measured. DI was calculated as follows: 


$$
\mathrm{DI}(\%)=\frac{\left(W-W_{1}\right)}{W} \times 100
$$

where $W$ is the initial weight of the granules (in this case, $W$ is $10 \mathrm{~g}$ ), and $W_{1}$ is the weight of the granules remaining on the dish.

Particle Size Distribution of Granules The particle size distribution was measured by sieving. Screens with mesh sizes of $45,75,150,250,355$ and $500 \mu \mathrm{m}$ were chosen for this study. These screens were piled one upon the other with the smaller mesh size screen placed at the lower position. The test granule $(5 \mathrm{~g})$ was placed on the top of the screen and then sieving was performed using a benchtop sieve shaker (M-3T; Tsutsui Scientific Instruments, Tokyo, Japan). Granules remaining on each screen were weighed and then $\mathrm{d} 10$, d50 and $\mathrm{d} 90$ were calculated. $\mathrm{d} 50$ is the median diameter, while $\mathrm{d} 10$ and $\mathrm{d} 90$ are for 10 and $90 \%$ of the particle size distribution. Using these granule size parameters, the span was calculated as follows:

$$
\text { Span }=\frac{d 90-d 10}{d 50}
$$

Tablet Weight Variation Tablet weight was measured and then the coefficient of variance $(\mathrm{CV})$ was calculated as follows:

$$
\mathrm{CV}=\sigma / \bar{x}
$$

where $\sigma$ is the standard deviation and $\bar{x}$ is the average tablet weight. The designated mini-tablet weight $(15 \mathrm{mg})$ was equivalent to $1 / 12$ of the regular tablet $(180 \mathrm{mg})$; thus, for the experiment to evaluate the relationships between granulation process variables and tablet weight variation, the gross weight of 12 mini-tablets was regarded as one piece of data for comparing the CV of mini-tablets with that of a regular tablet. The number of tablet weight data to calculate CV was 20 for both mini- and regular tablets. By contrast, for the experiment to examine the effect of the hole position of multiple-tip tooling on tablet weight variation, mini-tablets prepared from indi- vidual holes were separately sampled for 60 cycles and then the $\mathrm{CV}$ of the tablet weight corresponding to each hole was calculated.

Data Analysis The experimental data were analyzed with the statistical software JMP ${ }^{\circledR}$ Pro 15 (SAS Institute, Cary, NC, U.S.A.). Levels of significance were set at $p<0.05, p<0.01$, and $p<0.0001$.

\section{Results and Discussion}

DSD Analysis to Clarify the Effect of the Fluidized Bed Granulation Process on Granule Properties We first performed a screening study using DSD to investigate the effect of the granulation process on the powder properties of the resulting granules. The formulation and process variables that may affect the fluidized bed granulation were selected as evaluated factors as follows: the grinding speed of the sample mill in the process of grinding the API (X1), the MCC content in granules (X2), inlet air temperature (X3), binder concentration (X4) and the spray speed of binder solution (X5) in the granulation process. Before the screening study, the particle size of the ground API was measured. The d50 values steadily decreased with higher grinding speed: they were 33.6, 26.6 and $15.9 \mu \mathrm{m}$ after grinding at 7000,9000 and $11000 \mathrm{rpm}$, respectively (see Supplementary Material, Table s1). The test granules were prepared according to DSD, and then the powder properties of the resulting granules were measured (Table 1). After that, the regression models for each granule property were constructed by using effective design-based model selection for DSDs. ${ }^{8,24)}$ Regarding the experimental data for use in the analysis, the values of the evaluated factors were converted from practical conditions into coded ones (i.e., $-1,0$ or 1) to compare the relative effects of the factors on the granulation properties. Figure 1 shows the scatterplots of the experimental versus predicted values. In particular, the coefficients of determination adjusted by the degrees of freedom (adjusted $R^{2}$ ) of CI was extremely high, 0.97 , indicating that the vari-

\begin{tabular}{|c|c|c|c|c|c|c|c|c|c|c|c|c|}
\hline \multirow{3}{*}{$\begin{array}{c}\text { Granule } \\
\quad \#\end{array}$} & \multicolumn{5}{|c|}{ Factors } & \multicolumn{5}{|c|}{ Granule properties ${ }^{a)}$} & \multirow{2}{*}{\multicolumn{2}{|c|}{$\frac{\text { Tablet property }^{b)}}{\mathrm{CV}}$}} \\
\hline & \multirow{2}{*}{$\begin{array}{c}\mathrm{X} 1 \\
(\mathrm{rpm})\end{array}$} & \multirow{2}{*}{$\begin{array}{l}\mathrm{X} 2 \\
(\%)\end{array}$} & \multirow{2}{*}{$\begin{array}{c}\mathrm{X} 3 \\
\left({ }^{\circ} \mathrm{C}\right)\end{array}$} & \multirow[b]{2}{*}{$\begin{array}{l}\mathrm{X} 4 \\
(\%)\end{array}$} & \multirow{2}{*}{$\begin{array}{c}\mathrm{X} 5 \\
(\mathrm{~g} / \mathrm{min})\end{array}$} & \multirow[b]{2}{*}{$\mathrm{d} 50(\mu \mathrm{m})$} & \multirow[b]{2}{*}{ Span } & \multirow[b]{2}{*}{ DI $(\%)$} & \multirow[b]{2}{*}{$\operatorname{AR}\left({ }^{\circ}\right)$} & \multirow[b]{2}{*}{ CI $(\%)$} & & \\
\hline & & & & & & & & & & & $\begin{array}{l}\text { Mini } \\
\text { tablet }\end{array}$ & $\begin{array}{c}\text { Regular } \\
\text { tablet }\end{array}$ \\
\hline 1 & 9000 & 24 & 80 & 10 & 1.5 & $201 \pm 8.63$ & $1.59 \pm 0.11$ & $9.79 \pm 0.33$ & $47.7 \pm 1.04$ & $39.2 \pm 0.46$ & 0.0400 & 0.0079 \\
\hline 2 & 9000 & 34 & 60 & 4 & 2.5 & $146 \pm 2.95$ & $1.37 \pm 0.03$ & $22.40 \pm 4.76$ & $42.5 \pm 0.6$ & $26.0 \pm 0.17$ & 0.0426 & 0.0122 \\
\hline 3 & 7000 & 29 & 80 & 10 & 2.5 & $216 \pm 13.2$ & $1.40 \pm 0.05$ & $16.00 \pm 1.17$ & $44.7 \pm 0.42$ & $33.9 \pm 0.56$ & 0.0318 & 0.0143 \\
\hline 4 & 11000 & 29 & 60 & 4 & 1.5 & $147 \pm 1.83$ & $1.43 \pm 0.04$ & $24.20 \pm 2.92$ & $43.1 \pm 2.58$ & $28.1 \pm 0.94$ & 0.0498 & 0.0097 \\
\hline 5 & 11000 & 34 & 70 & 10 & 2.5 & $187 \pm 22.3$ & $1.63 \pm 0.31$ & $12.40 \pm 1.16$ & $47.0 \pm 2.09$ & $39.3 \pm 0.22$ & 0.0301 & 0.0189 \\
\hline 6 & 7000 & 24 & 70 & 4 & 1.5 & $169 \pm 6.73$ & $1.27 \pm 0.06$ & $26.20 \pm 3.65$ & $42.6 \pm 2.55$ & $27.8 \pm 0.76$ & 0.0429 & 0.0153 \\
\hline 7 & 11000 & 34 & 80 & 7 & 1.5 & $168 \pm 19.3$ & $1.27 \pm 0.08$ & $12.30 \pm 1.23$ & $43.8 \pm 1.92$ & $32.2 \pm 0.54$ & 0.0368 & 0.0106 \\
\hline 8 & 7000 & 24 & 60 & 7 & 2.5 & $210 \pm 8.02$ & $1.36 \pm 0.04$ & $21.40 \pm 5.04$ & $43.8 \pm 0.82$ & $27.5 \pm 0.27$ & 0.0316 & 0.0140 \\
\hline 9 & 7000 & 34 & 80 & 4 & 2.0 & $166 \pm 3.12$ & $1.33 \pm 0.01$ & $37.70 \pm 13.2$ & $42.9 \pm 2.12$ & $29.3 \pm 1.09$ & 0.0330 & 0.0064 \\
\hline 10 & 11000 & 24 & 60 & 10 & 2.0 & $252 \pm 49.6$ & $1.49 \pm 0.06$ & $6.77 \pm 0.99$ & $49.4 \pm 0.77$ & $39.1 \pm 1.19$ & 0.0516 & 0.0116 \\
\hline 11 & 11000 & 24 & 80 & 4 & 2.5 & $195 \pm 11.4$ & $1.26 \pm 0.05$ & $28.80 \pm 0.54$ & $42.8 \pm 0.92$ & $28.0 \pm 0.9$ & 0.0389 & 0.0118 \\
\hline 12 & 7000 & 34 & 60 & 10 & 1.5 & $179 \pm 16.7$ & $1.41 \pm 0.17$ & $11.70 \pm 0.66$ & $44.0 \pm 1.36$ & $34.7 \pm 1.18$ & 0.0341 & 0.0122 \\
\hline 13 & 9000 & 29 & 70 & 7 & 2.0 & $183 \pm 3.52$ & $1.39 \pm 0.07$ & $21.40 \pm 1.3$ & $43.0 \pm 1.48$ & $28.8 \pm 1.19$ & 0.0360 & 0.0145 \\
\hline
\end{tabular}
ance of CI was largely explained by the evaluated factors.

Table 1. Experimental Design for Formulation and Granulation Variables of the Model Granules and Properties of the Resulting Granules and Tablets

Test granules were prepared with different conditions according to definitive screening design (DSD). The evaluated factors assigned to DSD were: the grinding speed of the sample mill at the grinding process of the active pharmaceutical ingredient (X1), microcrystalline cellulose content in granules (X2), inlet air temperature (X3), binder concentration (X4) and the spray speed of the binder solution (X5) at the granulation process. $a$ ) Each value represents the mean \pm standard deviation $(\mathrm{S} . \mathrm{D}$.) $(N=3)$. $b)$ Each value represents the mean $(N=20)$. 

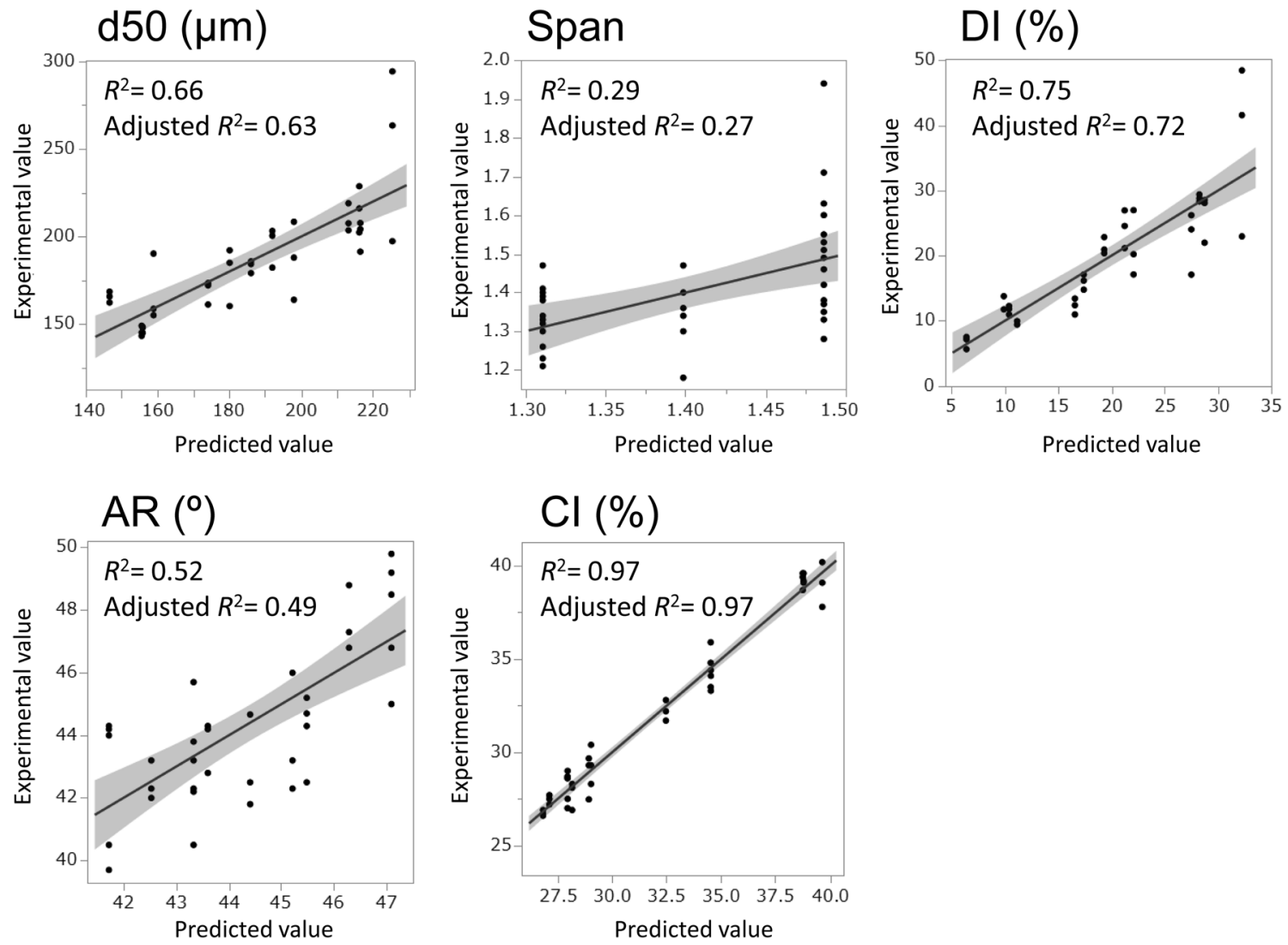

Fig. 1. Scatterplots of Experimental versus Predicted Values for Granule Properties Modeled Using DSD

$R^{2}$, the coefficient of determination; adjusted $R^{2}$, the coefficient of determination adjusted by the degrees of freedom. The shaded areas represent the $95 \%$ confidence intervals.

Based on the regression models, the crucial factors on each granule property were identified. X2, X4 and X5 were significant factors for d50 (Table 2), and X4 had a significant impact on the span (Table 3). DI was significantly affected by X1 and X4 (Table 4). For AR, X1 and X4 turned out to be significant (Table 5). X1, X3, X4 and X5 showed significant effects for CI (Table 6). In addition, two-factor interaction between X1 and $\mathrm{X} 4$ and several pure-quadratic effects (i.e., X3*X3 and $\mathrm{X} 4 * \mathrm{X} 4)$ were also significant for CI. The magnitude of the effect of the factors on each property can be understood by the $t$ - and estimate values: higher $t$ - and estimate values mean that the effect of the factor is more significant. Furthermore, the plus and minus signs of the estimates express the positive and negative effects of the factors. From the results, the mode of action of the crucial factors was fully characterized. In particular, X4 showed an extremely strong effect on all granule properties. Namely, a higher binder concentration in the binder solution produced granules having a larger d50 and span, lower DI, and higher AR and CI. An increase in d50 indicates that the granulation process was accelerated further. As far as the present study is concerned, the binder content in the resulting granules was set at the same level $(3.0 \mathrm{~g}$ of binder in $98 \mathrm{~g}$ of granules), and then a higher binder concentration means a smaller volume of binder solution to be sprayed on the powder during the fluidized bed granulation. Thus, the observed result can also be interpreted as a dense distribution of binder is more important for accelerating the granulation process rather than a large volume but low concentration. One possible mechanism for this is as follows: the harder granules were prepared by the thick binder solution and then they were difficult to crack, resulting in enlarging the granule size. In addition regarding $\mathrm{d} 50$, the effect of $\mathrm{X} 2$ was very large and it was almost equal to that of $\mathrm{X} 4$, indicating that the composition of granules also had a significant impact on the granulation process. As for DI, it decreased with a higher binder concentration, indicating the production of granules with less dispersion. This result seems to be reasonable because the dispersion of the granules generally decreased with enlarging the granule size. The increase in the span of the granules was probably caused by the smaller volume of binder solution. A smaller volume of binder solution could not be spread over the entire powder surface. As a consequence, many primary particles were left ungranulated, resulting in a wider size distribution of the granules. Regarding the AR and CI of the granules, they increased with a higher binder concentration. This means that the flowability of the granules became worse with continuing the granulation process. In most cases, fine particles exhibit poor flowability because of higher cohesive forces, which are due to the small size and large surface area, and then the flowability is improved by granulation. ${ }^{25,26)}$ Interestingly, the observed result was an unexpected one for us. The reason is probably due to the production of granules with irregular shapes. The flowability of granules is affected by a wide variety of powder properties, as well as particle size; the shape of the granule is also regarded as being an important factor for flowability. ${ }^{27)}$ Thus, we thought that the irregularshaped granules with wide size distribution were produced by the fluidized bed granulation, and then this property led to 
Table 2. Regression Model for d50 Estimated Using DSD

\begin{tabular}{lrrr}
\hline \hline & Estimate & Standard error & $t$-Value \\
\hline Intercept & 186.04 & 3.138 & 59.28 \\
X2 (MCC content in granule) & -18.19 & 3.578 & -5.08 \\
X4 (Binder concentration) & 21.20 & 3.578 & 5.92 \\
X5 (Spray speed of binder solution) & 8.95 & 3.578 & $<0.0001^{* * *}$ \\
\hline
\end{tabular}

Table 3. Regression Model for Span Estimated Using DSD

\begin{tabular}{lcccc}
\hline \hline & Estimate & Standard error & \multicolumn{1}{c}{$t$-Value } & \\
\hline Intercept & 1.398 & 0.020 & 70.293 \\
X4 (Binder concentration) & 0.088 & 0.023 & 3.865 \\
\hline
\end{tabular}

Table 4. Regression Model for DI Estimated Using DSD

\begin{tabular}{lccc}
\hline \hline & Estimate & Standard error & $t$-Value \\
\hline Intercept & 19.32 & 0.786 & 24.57 \\
X1 (Grinding speed of API) & -2.85 & 0.897 & -3.18 \\
X3 (Inlet air temperature) & 1.80 & 0.897 & $0.0001 * * *$ \\
X4 (Binder concentration) & -8.26 & 0.097 & $0.032 * *$ \\
X5 (Spray speed of binder solution) & 1.70 & 0.897 & -9.22 \\
\hline
\end{tabular}

Table 5. Regression Model for AR Estimated Using DSD

\begin{tabular}{|c|c|c|c|c|}
\hline & Estimate & Standard error & $t$-Value & $p$-Value \\
\hline Intercept & 44.41 & 0.288 & 154.16 & $<0.0001 * * *$ \\
\hline X1 (Grinding speed of API) & 0.80 & 0.328 & 2.45 & $0.0195 *$ \\
\hline X4 (Binder concentration) & 1.88 & 0.328 & 5.71 & $<0.0001 * * *$ \\
\hline
\end{tabular}

Table 6. Regression Model for CI Estimated Using DSD

\begin{tabular}{|c|c|c|c|c|}
\hline & Estimate & Standard error & $t$-Value & $p$-Value \\
\hline Intercept & 28.93 & 0.376 & 76.97 & $<0.0001 * * *$ \\
\hline X1 (Grinding speed of API) & 1.34 & 0.155 & 8.64 & $<0.0001 * * *$ \\
\hline X3 (Inlet air temperature) & 0.67 & 0.155 & 4.29 & $0.0002 * *$ \\
\hline X4 (Binder concentration) & 4.63 & 0.155 & 29.79 & $<0.0001 * * *$ \\
\hline X5 (Spray speed of binder solution) & -0.66 & 0.155 & -4.27 & $0.0002 * *$ \\
\hline $\mathrm{X} 1 * \mathrm{X} 4$ & 1.55 & 0.197 & 7.87 & $<0.0001 * * *$ \\
\hline $\mathrm{X} 3 * \mathrm{X} 3$ & 0.86 & 0.370 & 2.33 & $0.0263^{*}$ \\
\hline $\mathrm{X} 4 * \mathrm{X} 4$ & 3.00 & 0.327 & 9.16 & $<0.0001 * * *$ \\
\hline
\end{tabular}

lower flowability. In sum, the screening study with DSD succeeded in characterizing the relationships between formulation and process variables in the granulation process and the powder properties of the resulting granules.

DSD is a new class of three-level experimental design and is excellent for screening crucial factors. ${ }^{7,8)}$ To create the experimental design, three levels of factors are assigned by the conference matrix. The conference matrix is composed of fold-over pairs corresponding to each evaluated factor and overall center points. Thus, the number of experimental runs resulted in just more than twice the number of evaluated factors. The data analysis procedures of DSD are divided into two steps. ${ }^{8,24)}$ In the first step, the DSD analysis is dedicated to the evaluation of the main effects of the factors. The second step focuses on the factors that are found to be significant in the first step and then evaluates their two-factor interactions and quadratic effects. The DSD has been developed based on the concept of the sparsity-of-effects principle: namely, in most cases, although a large number of factors are contained in the dataset, only a limited number of factors are expected to affect the response. The main effects are always orthogonal to the two-factor interactions and quadratic effects, while two-factor interactions and quadratic effects are partially confounded with each other. ${ }^{8)}$

DSD has been recently applied to several pharmaceutical formulation studies. The dosage forms to be evaluated covered a wide range (e.g., oral disintegrating films, ${ }^{9}$ ) cross-linked chitosan disk-modified drug release, ${ }^{10)}$ intranasal transferosomal mucoadhesive gel formulation, ${ }^{11)}$ ointment formulation ${ }^{12)}$ and monoclonal antibody drug products. ${ }^{14)}$ ). DSD enables the screening of crucial factors from a large number of potential factors with a small number of experimental runs: thus, it 
Mini-tablet

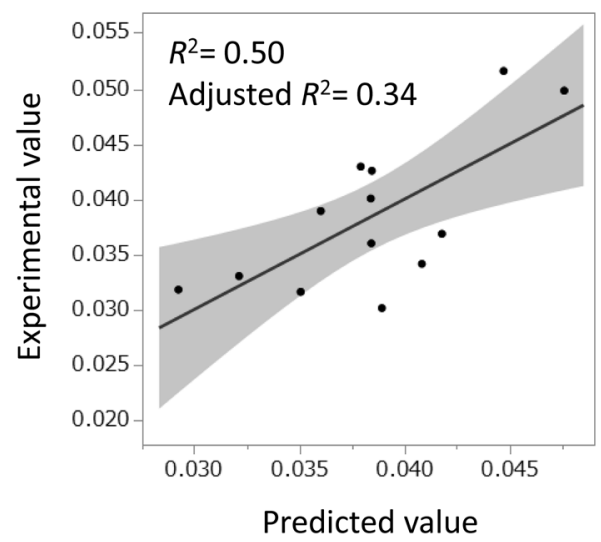

Regular tablet

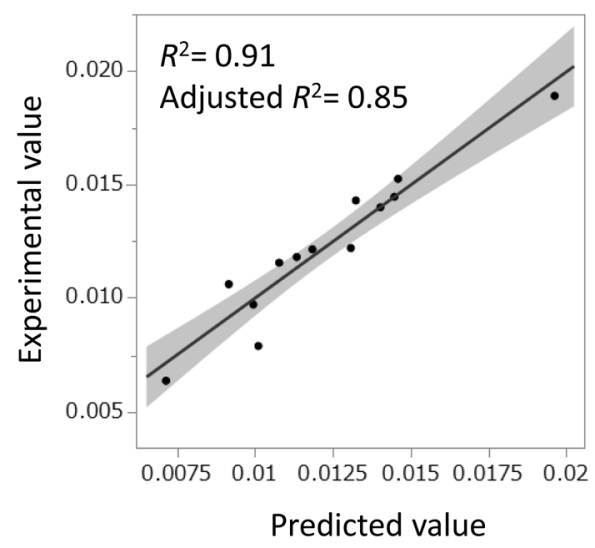

Fig. 2. Scatterplots of Experimental versus Predicted Values for the CV of Mini- and Regular Tablets Modeled Using DSD

$R^{2}$, the coefficient of determination; adjusted $R^{2}$, the coefficient of determination adjusted by the degrees of freedom. The shaded areas represent the $95 \%$ confidence intervals.

Table 7. Regression Model for the CV of Mini-tablets Estimated Using DSD

\begin{tabular}{|c|c|c|c|c|}
\hline & Estimate & Standard error & $t$-Value & $p$-Value \\
\hline Intercept & 0.0384 & 0.0015 & 24.91 & $<0.0001 * * *$ \\
\hline X1 (Grinding speed of API) & 0.0034 & 0.0018 & 1.93 & 0.0861 \\
\hline X3 (Inlet air temperature) & -0.0029 & 0.0018 & -1.66 & 0.1323 \\
\hline X5 (Spray speed of binder solution) & -0.0029 & 0.0018 & -1.64 & 0.1353 \\
\hline
\end{tabular}

Table 8. Regression Model for the CV of Regular Tablets Estimated Using DSD

\begin{tabular}{|c|c|c|c|c|}
\hline & Estimate & Standard error & $t$-Value & $p$-Value \\
\hline Intercept & 0.0145 & 0.0009 & 15.54 & $<0.0001 * * *$ \\
\hline X3 (Inlet air temperature) & -0.0009 & 0.0004 & -2.14 & 0.0697 \\
\hline X4 (Binder concentration) & 0.0009 & 0.0004 & 2.34 & 0.0520 \\
\hline X5 (Spray speed of binder solution) & 0.0016 & 0.0004 & 3.86 & $0.0062 * *$ \\
\hline $\mathrm{X} 3 * \mathrm{X} 3$ & -0.0055 & 0.0008 & -6.47 & $<0.0001 * * *$ \\
\hline $\mathrm{X} 5 * \mathrm{X} 5$ & 0.0026 & 0.0008 & 3.09 & $0.0176^{*}$ \\
\hline
\end{tabular}

could become a powerful tool for designing formulations of pharmaceuticals.

Understanding the Manufacturing Process Variables for Tablet Weight Variation In the next phase of the present study, we investigated the tablet weight variation of the miniand regular tablets. This experiment used the $\mathrm{CV}$ of tablet weight as an index of the tablet weight variation. As mentioned in Experimental, they were calculated from 20 data for each tablet. The designated weight of the mini-tablet was set at $1 / 12$ that of a regular tablet. To eliminate the effect caused by the difference in tablet weight from the calculated $\mathrm{CV}$, the weight of 12 tablets was regarded as one piece of data for the mini-tablet, whereas the weight of one tablet was used as one piece of data for a regular tablet. As shown in Table 1, the CVs of mini-tablets ranging from 0.0301 to 0.0516 were larger than those of regular tablets, ranging from 0.0064 to 0.0189 . The obtained experimental data were analyzed with DSD, and then regression models for both tablets were constructed between the formulation and process variables in the granulation process and the CVs. The scatterplots of experimental versus predicted values for the $\mathrm{CV}$ of mini- and regular tablets are shown in Fig. 2. The resulting regression model for the regular tablet was meaningful $(p=0.0015)$, whereas for the mini-tablet, no significant meaning was observed $(p=0.0849)$. Additionally, the adjusted $R^{2}$ value for the regression model for the regular tablet, 0.85 , was much higher than that for the mini-tablet, 0.34 . These issues indicate that for the mini-tablet, it is more difficult to model the tablet weight variation by the evaluated factors. As for the crucial factors, the DSD analysis did not show any significant factors for the CV of the minitablet (Table 7). By contrast, several significant factors were identified from the regular tablet (Table 8). They include the main effect of X5 and the quadratic effects of X3 and X5: namely, the regular tablets were produced with a higher $\mathrm{CV}$ from the granules prepared with a lower inlet air temperature and a faster spray speed of the binder solution. We think that these crucial factors certainly affected the tablet weight variation through modifying granule properties; however, it was hard to gather a further understanding of the relationships between granule properties and the $\mathrm{CV}$ from the data because the effects of $\mathrm{X} 3$ and $\mathrm{X} 5$ on granule properties were very slight compared with those of the other factors such as $\mathrm{X} 4$ (Tables 2-6).

Subsequently, we took a closer look at individual data. The 
regular tablets having the largest and smallest $\mathrm{CV}$ were produced from granules $\# 5$ and $\# 9$, respectively (Table 1). Their granule properties were distinct from each other. For example, granule \#5 had poorer flowability than the others because their AR and CI were almost the highest, whereas granule \#9 possessed better flowability because of the relatively low AR and CI. In addition, granule \#5 showed the largest span among the test granules. From these issues, flowability was likely to be the crucial granule property for the tablet weight variation. The flowability of granules is regarded as being important for die-filling performance. This subject has been extensively investigated..$^{9,25,28,29)}$ These studies reported that powders with poorer flowability are accompanied by an increase in tablet weight variation.

As for the CVs of mini-tablets, the changed behavior relative to the difference in granules appeared to be independent from that of regular tablets. The present study did not show any affecting factors for mini-tablets. By contrast, to date, several studies have reported that several powder properties, mainly flowability, have a significant impact on the mini-tablet weight variation. ${ }^{19-21)}$ These previous studies have tested sample powders having a wider range of powder properties compared with the granules used in the present study, resulting in the detectable effect on the powder properties. We also think that other process variables, other than the granulation process, significantly affected the tablet weight variation. To support this speculation, several technical reports investigated the influence of process parameters concerning the tableting pro- cess on die-filling performance and tablet weight variation for manufacturing mini-tablets. They include the following: the disk rotational speed of the rotary tableting machine, ${ }^{30,31)}$ fill cam type, ${ }^{31)}$ fill depth, ${ }^{31)}$ feed paddle design, ${ }^{32)}$ paddle speed ${ }^{32)}$ and the die-hole position of multiple-tip tooling. ${ }^{30)}$ Among them, the hole position of multiple-tip tooling may well affect the tablet weight variation of our mini-tablets. If the weight of the mini-tablets would substantially fluctuate by the difference in the die-hole position, it is reasonable that no crucial factors would have been observed from this study (Table 7). Based on this perspective, this study further investigated the $\mathrm{CV}$ of mini-tablets produced from different die holes.

The multiple-tip tooling used in this study had six holes arranged in a circle on the die (Fig. 3a). To conduct this experiment, individual holes were numbered and then mini-tablets produced from each hole were separately sampled. Three different granules (\#5, \#6 and \#10) were used for this experiment. As shown in Table 1, granules \#5 and \#10 resulted in mini-tablets having the lowest and highest $\mathrm{CV}$ values $(0.0301$ and 0.0516) among the test granules, whereas granule \#6 produced the mini-tablet with middle $\mathrm{CV}, 0.0429$. The mean tablet weight and CV produced from individual die holes are shown in Figs. $3 b$ and c. These values were calculated from 60 tablets. From Fig. 3b, there was no obvious tendency in the effect of the hole position on tablet weight. In brief, in the case of the mini-tablet produced of granule \#6, heavier tablets were produced from holes \#1 and \#2, but this tendency was not observed for other mini-tablets. By contrast, the $\mathrm{CV}$
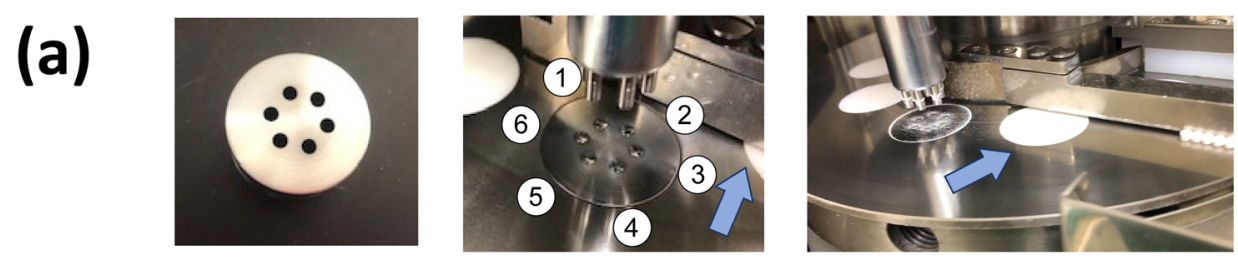

(b)
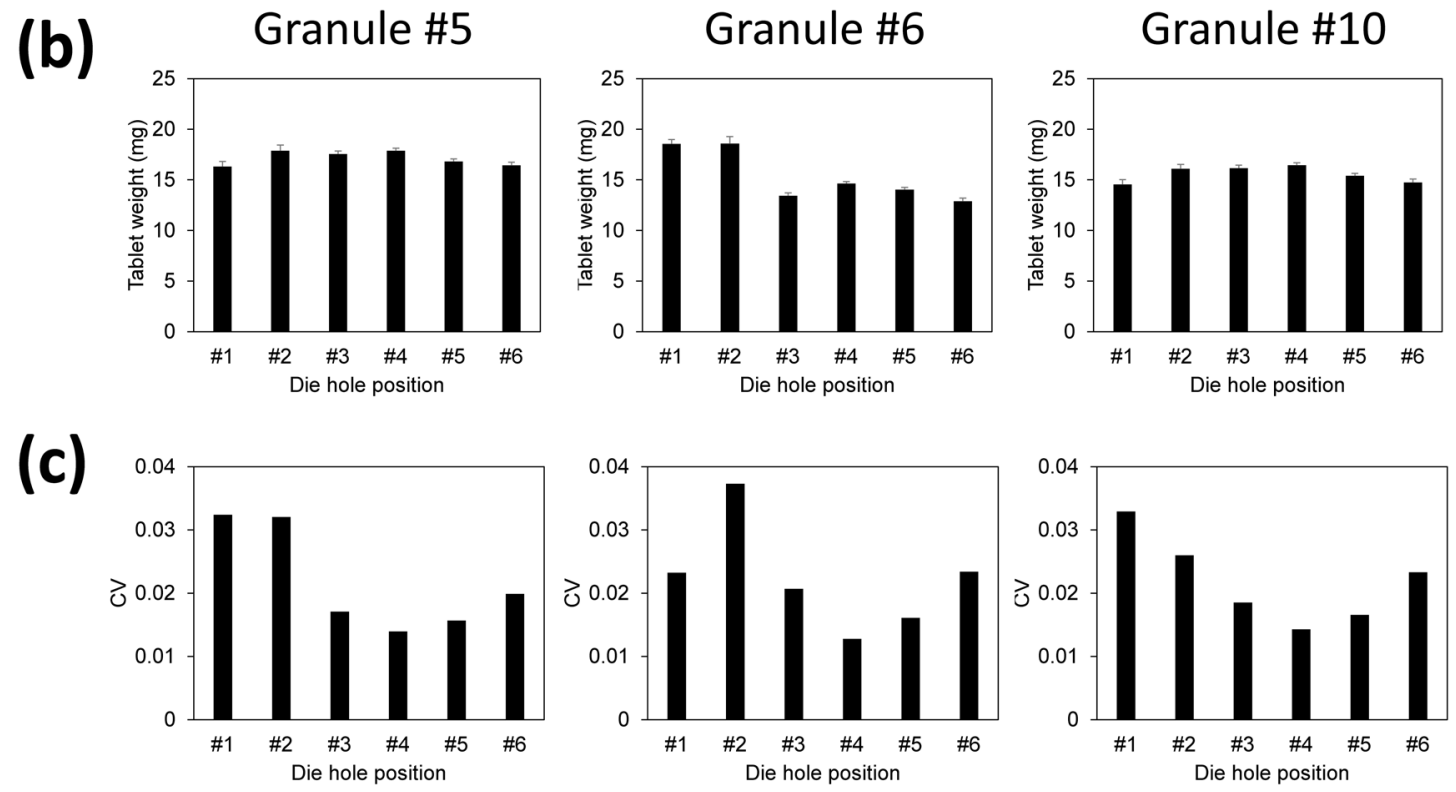

Fig. 3. Weight Variation of Mini-tablets Prepared from Various Die Holes of Multiple-Tip Tooling

(a) Die-hole position of multiple-tip tooling. Arrow shows rotational direction of the rotary disk. Holes \#1 and \#2 are located in front of the rotational direction, whereas holes \#4 and \#5 are located behind. The mean (b) and CV (c) of tablet weight prepared from individual die holes. The tablet weights represent the mean \pm S.D. $(N=60)$. The CV values were calculated from 60 tablets. (Color figure can be accessed in the online version.) 
Table 9. Correlation Model for the CV of the Mini-tablets Estimated Using ANOVA

\begin{tabular}{lrrrc}
\hline \hline & Estimate & Standard error & $t$-Value & $p$-Value \\
\hline Intercept & 0.0220 & 0.0009 & 25.04 & $<0.0001^{* * *}$ \\
Granule \#5 & -0.0002 & 0.0012 & -0.13 & 0.8993 \\
Granule \#6 & 0.0002 & 0.0012 & 0.19 & 0.8500 \\
Granule \#10 & -0.0001 & 0.0012 & -0.06 & 0.9500 \\
Hole\#1 & 0.0075 & 0.0020 & 3.82 & $0.0034^{* *}$ \\
Hole\#2 & 0.0098 & 0.0020 & 4.97 & $0.0006^{* *}$ \\
Hole\#3 & -0.0032 & 0.0020 & -1.65 & 0.1304 \\
Hole\#4 & -0.0083 & 0.0020 & -4.25 & $0.0017^{* *}$ \\
Hole\#5 & -0.0059 & 0.0020 & -3.00 & $0.0134^{*}$ \\
Hole\#6 & 0.0002 & 0.0020 & 0.10 & 0.9229 \\
\hline
\end{tabular}

showed some association with hole position (Fig. 3c). Overall, the mini-tablets prepared from holes \#1 and \#2 showed a larger CV than those prepared by holes \#4 and \#5. To identify this issue more clearly, statistical analysis was performed. The $\mathrm{CV}$ data for the mini-tablets produced from the three different granules were merged and were analyzed using ANOVA. As anticipated, the effect of the hole position was confirmed to be significant, whereas the difference in granules did not have a significant effect on the CV (see Supplementary Material, Table s2). Furthermore, the mode of action of individual hole positions was identified from the correlation model (Table 9). From the estimated values, it was confirmed that the $\mathrm{CV}$ values of mini-tablets produced by holes $\# 1$ and $\# 2$ were significantly higher, while those by holes \#4 and \#5 were significantly lower. Holes \#1 and \#2 are located in front of the rotational direction of the rotary disk, whereas holes \#4 and \#5 are located behind (Fig. 3a). A similar result has been reported recently by Kurashima et $a l .^{30}$ ) The authors manufactured 2.3-mm tablets using a rotary tableting machine with 12 tips tooling at a rotational speed ranging from 20 to $60 \mathrm{rpm}$. The tableting machine was stopped at the designated cycles of the tableting, and then the mini-tablets were sampled separately from the individual holes. As a consequence, they observed that mini-tablets with a higher weight variation were produced by the die holes located in the out and forward direction relative to the rotating disk regardless of powders and the type of feeder systems. Although further investigation is required, the die-hole position of multiple-tip tooling is thought to be a crucial element for tablet weight variation in manufacturing mini-tablets. We expect that the optimization of die-hole arrangement will become an important research subject to establish a robust manufacturing method for mini-tablets that could ensure tablet weight uniformity.

\section{Conclusion}

This study investigated the relationships between process variables of fluidized bed granulation, granule properties and the mini-tablet weight variation. To achieve this research subject, screening with DSD was used. Owing to the new screening method, we efficiently characterized crucial factors with a small amount of experimental data. In the initial phase of this study, the mode of action of formulation and process valuables relating to the granulation process on granule properties was fully clarified. In the next phase, we examined the effect of the evaluated factors on tablet weight variation. As a result, the relationships between granule properties and tablet weight variation were ambiguous and it was hard to gain a comprehensive understanding of this issue. We further considered other manufacturing variables factors besides the granulation process. Consequently, it was confirmed that that the die-hole position of the multiple-tip tooling used for manufacturing the minitablets has a significant impact on the $\mathrm{CV}$ of the resulting minitablet, and the effect of the die-hole position on tablet weight variation was much more significant than that of granule properties. This would provide valuable information for designing mini-tablet products with good tablet weight uniformity.

Acknowledgments The authors thank Pharmaceutical Department, Watahan Trading Co., Ltd. for a generous donation of Acetaminophen. This work was supported by the Japan Society for the Promotion of Science (JSPS) KAKENHI [Grant numbers 20K06986 and 20K15970].

Conflict of Interest The authors declare no conflict of interest. The Laboratory of Pharmaceutical Technology, University of Toyama is an endowed department, supported by an unrestricted Grant from the Nichi-Iko Pharmaceutical Co., Ltd. (Toyama, Japan).

Supplementary Materials The online version of this article contains supplementary materials.

\section{References}

1) Tawfeek H. M., Saleem I. Y., Roberts M., J. Pharm. Sci., 103, 2470-2483 (2014).

2) Thabet Y., Klingmann V., Breitkreutz J., J. Clin. Pharmacol., 58 (Suppl 10), s26-s35 (2018).

3) Klingmann V., Spomer N., Lerch C., Stoltenberg I., Frömke C., Bosse H. M., Breitkreutz J., Meissner T., J. Pediatr., 163, 1728-1732 (2013).

4) Yu L. X., Amidon G., Khan M. A., Hoag S. W., Polli J., Raju G. K., Woodcock J., AAPS J., 16, 771-783 (2014).

5) Yu L. X., Pharm. Res., 25, 781-791 (2008).

6) International Conference on Harmonization of Technical Requirements for Registration of Pharmaceuticals for Human Use, Pharmaceutical Development-Q8 (R2), 2009.

7) Jones B., Nachtsheim C. J., J. Qual. Technol., 43, 1-15 (2011).

8) "JMP15 Design of Experiments Guide," SAS Institute, Cary, NC, U.S.A., 2020.

9) Mazumder S., Pavurala N., Manda P., Xu X., Cruz C. N., Krishnaiah Y. S. R., Int. J. Pharm., 527, 151-160 (2017).

10) Gabbay R. S., Kenett R. S., Scaffaro R., Rubinstein A., Eur. J. Pharm. Sci., 125, 102-109 (2018).

11) Salem H. F., Kharshoum R. M., Abou-Taleb H. A., Naguib D. M. AAPS PharmSciTech, 20, 181 (2019).

12) Dong Y., Qu H., Pavurala N., Wang J., Sekar V., Martinez M. N., Fahmy R., Ashraf M., Cruz C. N., Xu X., Int. J. Pharm., 544, 254-264 (2018).

13) van Heugten A. J. P., Vromans H., AAPS PharmSciTech, 19, 2330 2334 (2018).

14) Goldman J. M., More H. T., Yee O., Borgeson E., Remy B., Rowe J., Sadineni V., J. Pharm. Sci., 107, 2592-2600 (2018).

15) Schinzinger O., Schmidt P. C., Pharm. Dev. Technol., 10, 175-188 (2005).

16) Petrović J., Chansanroj K., Meier B., Ibrić S., Betz G., Eur. J. Pharm. Sci., 44, 227-234 (2011).

17) Arndt O. R., Baggio R., Adam A. K., Harting J., Franceschinis E., Kleinebudde P., J. Pharm. Sci., 107, 3143-3152 (2018).

18) Gabbott I. P., Al Husban F., Reynolds G. K., Eur. J. Pharm. Bio- 
pharm., 106, 70-78 (2016)

19) Goh H. P., Heng P. W. S., Liew C. V., Int. J. Pharm., 534, 279-286 (2017).

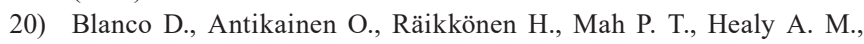
Juppo A. M., Yliruusi J., Int. J. Pharm., 581, 119280 (2020).

21) Zhao J., Yin D., Rowe J., Badawy S., Nikfar F., Pandey P., J. Pharm. Sci., 107, 1204-1208 (2018).

22) Hayashi Y., Tsuji T., Shirotori K., Oishi T., Kosugi A., Kumada S., Hirai D., Takayama K., Onuki Y., Int. J. Pharm., 532, 82-89 (2017).

23) Hayashi Y., Shirotori K., Kosugi A., Kumada S., Leong K. H., Okada K., Onuki Y., Pharmaceutics, 12, 601 (2020).

24) Jones B., Nachtsheim C. J., Technometrics, 59, 319-329 (2017).

25) Schiano S., Chen L., Wu C. Y., Powder Technol., 337, 78-83 (2018).
26) Xie X., Puri V. M., Adv. Powder Technol., 23, 1-7 (2012).

27) Sandler N., Wilson D., J. Pharm. Sci., 99, 958-968 (2010).

28) Sinka I. C., Schneider L. C., Cocks A. C., Int. J. Pharm., 280, 27-38 (2004).

29) Kachrimanis K., Petrides M., Malamataris S., Int. J. Pharm., 303, $72-80$ (2005).

30) Kurashima H., Uchida S., Kashiwagura Y., Tanaka S., Namiki N., Chem. Pharm. Bull., 68, 981-988 (2020).

31) Cho C. H., Kim J. Y., Park E. S., Powder Technol., 362, 90-100 (2020).

32) Goh H. P., Sia Heng P. W., Liew C. V., J. Pharm. Sci., 108, 11611171 (2019). 